\title{
Global English? Die Sprachkompetenz von Unternehmen und die Fremdsprachenausbildung
}

\author{
Dr. Greg Bond, John O’Donoghue (M.A.)
}

Die Grenzen unserer Sprache sind die Grenzen unserer Welt. Ludwig Wittgenstein

\section{Sprache im internationalen Kontext}

Als der US-Präsident John F. Kennedy am 26. Juni 1963 vor dem Schöneberger Rathaus in West-Berlin seine Solidarität mit der Stadt und ihren Menschen bekundete, sprach er natïrlich seine Muttersprache. Die Selbstverständlichkeit, mit der angenommen wird, daß die englische Sprache die internationale Sprache schlechthin sei, ist heute größer denn je. Aber Präsident Kennedy machte eine wichtige Ausnahme zu dieser Regel, die als einziger Satz von damals uns heute noch in Erinnerung bleibt. „Ich bin ein Berliner“, sagte er in grammatikalisch nicht ganz einwandfreiem Deutsch.

Im internationalen Kontext ohne Englisch auszukommen, ist heute fast unvorstellbar, aber es bleibt ebenso wichtig und wird noch wichtiger, Kenntnisse anderer Sprachen zu pflegen. Wünschenswert wird es immer sein, die andere Sprache so gut zu beherrschen, daß man mit den Menschen und eventuellen Geschäftspartnern sprechen und korrespondieren kann. Die Fähigkeit, erste Begrüßungen, allgemeine Höflichkeitsformeln und andere einfache Gepflogenheiten des Alltags in der Fremdsprache zu bewältigen, sollte sich jeder, der im Ausland tätig werden will, aneignen. Der gute Eindruck, den es macht, wenn man Menschen eines anderen Landes und einer anderen Kultur in ihrer eigenen Sprache begegnet, kann nicht unterschätzt werden. Solche einfachen Gesten des Respekts schon bei der ersten Kontaktaufnahme können in ihrer relativen Tragweite genauso werbewirksam sein wie die Worte John F. Kennedys. Zur Weltsprache Englisch kann und wird man dann sowieso meistens greifen, wenn es ins Detail geht - siehe John F. Kennedy.

Wer gut Russisch oder Chinesisch beherrscht und mit Russen oder Chinesen zusammenarbeiten will, wird sicherlich einen Vorteil haben. Die allermeisten Unternehmen aber, die international tätig sind oder sein wollen, werden auf Englisch angewiesen sein. $80 \%$ der Korrespondenz mit dem Ausland, sagten deutsche Unternehmen, wird auf Englisch abgewickelt (bfai-Informationen, zitiert in Wonneberger/Könecke, 1997, S. 7). Es ist zu vermuten, daß in den übrigen $20 \%$ ein guter Anteil deutscher Sprache steckt. Aber was bedeutet es, wenn Englisch die Weltsprache ist, ohne die man im internationalen Wettbewerb kaum bestehen kann? Und was bedeutet es, daß niemand es John F. Kennedy übelnahm, daß er in seinem deutschen Satz möglicherweise ein Wort zu viel benutzte? Und wie sieht es etwas genauer aus mit der globalen Sprache Englisch?

\section{Englisch als Weltsprache}

"Money speaks sense in a language all nations understand", schrieb die englische Schriftstellerin Aphra Behn 1677. Wenn das noch zutrifft, dann ist diese Sprache nicht das Geld alleine, sondern die englische Sprache. Und auch da müßte Aphra Behns satirisches Wort neu geschrieben werden, weil Englisch schon lange nicht mehr nur eine Sprache ist.

Daß Englisch heute als Weltsprache gilt, hat historische Ursachen. Im neunzehnten Jahrhundert war Englisch die Sprache der größten Kolonialmacht der Welt, die nicht nur ihre Sprache, sondern auch ihre eigene Kultur auf ihr Herrschaftsgebiet übertragen wissen wollte. Davon ist die Verbreitung der englischen Sprache in großen Teilen der Welt übriggeblieben, aber nun in einem multikulturellen Kontext, der zur Folge hat, daß es schon lange keine einzige Sprache mit dem Namen Englisch mehr gibt. Es gibt nicht eine Muttersprache Englisch, sondern viele Sprachen mit diesem Namen, jede mit eigenen lexikalischen und grammatischen Eigenheiten und voneinander stark divergierenden Aussprachen. Und jede dieser Sprachen steht gleichberechtigt neben den anderen.

Seit 1945 ist die Bedeutung der USA in internationaler Politik und Wirtschaft kontinuierlich gewachsen. Das ist der Grund, warum, nachdem Großbritannien längst keine Weltmacht mehr ist, der Einfluß der englischen Sprache sich weiterhin weltweit ausdehnt. Dazu kommt die in den vergangenen Jahrzehnten kontinuierlich gewachsene Bedeutung von Weltorganisationen wie den Vereinten Nationen, dem Internationalen Währungsfonds oder der Weltbank, die alle das Einflußgebiet der englischen Sprache mit erweitert haben. Schon 1898 erkannte Otto von Bismarck, welche Sprache am Ende des Jahrtausends die wichtigste sein würde. Gefragt, was die entscheidende Tatsache für die moderne Geschichte sei, soll er geantwortet haben, daß die Nordamerikaner Englisch reden (The Economist, 21.12.1996-3.1.1997, S. 45). Die immer intensiver und extensiver werdende Globalisierung unserer Wirtschaft und Kommunikationsmittel führt zu einer globalen Wahrnehmung der Welt - jedenfalls in Ansätzen. Diese globale Wahrnehmung läuft größtenteils über die englische Sprache. David Graddol (Graddol, S. 8) führt die wichtigsten internationalen Domänen von Englisch wie folgt an:

1. Working language of international organisations and conferences.

2. Scientific publication. 
3. International banking, economics, affairs and trade.

4. Advertising for global brands.

5. Audio-visual cultural products (e.g. film, TV, popular music).

6. International tourism.

7. Tertiary education.

8. International safety (e.g. "airspeak", "seaspeak").

9. International law.

10. As a "relay language" in interpretation and translation.

11. Technology transfer.

12. Internet communication.

Konkret heißt es für die Wirtschaft: die Sprache der internationalen Kommunikation ist zur Zeit Englisch, und sie wird es auf lange Zeit bleiben. Ohne Englisch in die Welt zu schauen, oder in der Welt tätig sein zu wollen, ist heute wie taubstumm sein. In vielen Bereichen (Tourismus, Flugverkehr, Marketing, Wissenschaften) werden die Geschäfte international nur oder überwiegend in englischer Sprache abgewickelt. $80 \%$ des auf Computer gespeicherten Wissens kann man nur auf Englisch lesen. Im Internet zeichnet sich eine Entwicklung ab, die nur auf den ersten Blick widerläufig zu sein scheint. Vor drei Jahren waren $98 \%$ aller Websites in Englisch, jetzt sind es nur noch $82 \%$. Das bedeutet aber nicht, daß die englische Sprache weniger bedeutend wird, sondern daß die rasante Ausbreitung des Netztes von einem proportional größeren Zuwachs an anderen Sprachen begleitet wird (The Economist, 18.-24.10.1997, S. 107-108).

Nach den Schätzungen von David Crystal gibt es derzeit 1.989 Millionen Menschen, die tagtäglich mit Englisch zu tun haben. Das ist ein Drittel der Weltbevölkerung. Die Zahl der Muttersprachler hingegen beläuft sich auf nur 377 Millionen (Crystal, 1995, S. 108). Es ist sinnvoll, die englischsprechende Weltbevölkerung in drei Gruppen einzuteilen: Muttersprachler; Menschen, für die Englisch die zweite Sprache ist, die sie fast wie eine Muttersprache beherrschen; und Menschen, die Englisch als Fremdsprache sprechen. Laut Graddol gibt es derzeit 375 Millionen Muttersprachler (70\% leben in den USA und Großbritannien), 375 Millionen Menschen, für die Englisch die zweite Sprache ist, und 750 Millionen, die Englisch als Fremdsprache benutzen (Graddol, S.10). Länder, deren Bevölkerung auf dem Weg ist, Englisch nicht nur als Fremdsprache, sondern als zweite Sprache zu beherrschen, sind unter vielen anderen: Argentinien, Dänemark, Libanon, Nicaragua, Norwegen, Schweden, und die Schweiz (Graddol, S. 11). Die Bundesrepublik Deutschland ist deswegen nicht dabei, weil die deutsche Sprache zumindest innerhalb Europas noch einen beträchtlichen wirtschaftlichen Einflußbereich hat.

Aber auch in Deutschland ist die Bedeutung von Englisch enorm. Betriebswirtschaft zu studieren heißt in vielen Ländern, gleichzeitig Englisch zu studieren. In der Bundesrepublik Deutschland wird von Studenten der Betriebswirtschaft zu Recht erwartet, daß sie die englische Sprache gut beherrschen. Eine Umfrage unter deutschen Wissenschaftlern ergab, daß $98 \%$ der Physi- ker Englisch als ihre tatsächliche Arbeitssprache betrachten. In allen Naturwissenschaften war diese Zahl sehr hoch, und bei Wirtschaftswissenschaftlem gaben $48 \%$ an, daß Englisch ihre Arbeitssprache ist (Graddol, S. 9). Die Präsenz der englischen Sprache in Deutschland geht weit über die verbreitete Verwendung zahlreicher internationaler Begriffe wie Team, Bestseller, High-tech, Recycling, City und viele andere hinaus. Englisch ist für viele Menschen ein tägliches Arbeitsinstrument.

Was die längerfristige Entwicklung der Sprachen auf unserem Globus in den nächsten fünfzig Jahren betrifft, so gehen Prognosen davon aus, daß andere Sprachen als Englisch in größeren regionalen Netzen und Handelsblöcken die Rolle einer örtlichen Lingua franca übernehmen könnten. Mit dem Ausbau der Wirtschaft und der Kommunikationsstrukturen in Asien und insbesondere in China könnte Mandarin diese Rolle für Ostasien übernehmen. Die „großen Sprachen“, laut Graddol, werden Chinesisch, Hindi/Urdu, Englisch, Spanisch und Arabisch sein (Graddol, S. 59). Die deutsche Sprache wird für Mitteleuropa ihre Bedeutung behalten. Es wird eine Situation entstehen, in der in unserer internationalisierten Welt die meisten Menschen mindestens zwei, wenn nicht gleich drei Sprachen beherrschen werden. Das sind die Sprache der regionalen Wirtschaft, die Sprache, die noch zu Hause benutzt wird, und Englisch, die als einzig wirklich internationale Sprache ihre Bedeutung behalten wird. Aussterben werden im Laufe dieser Entwicklung unzählige „kleinere“ Sprachen: ungefähr die Hälfte aller Sprachen der Welt werden zur Zeit in der Asien-Pazifik-Region gesprochen, und es wird erwartet, daß die Mehrzehl dieser Sprachen im Zuge der rasanten Entwicklung im nächsten Jahrhundert verlorengeht (Graddol, S. 38). Eins steht außer Zweifel, wie Graddol schreibt: "There is no reason to believe that any other language will appear in the next 50 years to replace English as the global lingua franca" (S. 58).

Daß Englisch als Mittel der internationalen Kommunikation unersetzlich ist, sollte als Chance und nicht als Hindernis verstanden werden. Es ist leicht möglich, Englisch soweit zu beherrschen, daß man international kommunizieren kann. Denn es geht vorrangig nicht darum, das sprachliche Niveau eines Muttersprachlers zu erreichen. Die Statistiken zeigen es. Muttersprachler werden die globale Zukunft der englischen Sprache nicht wesentlich bestimmen. Es geht um die Vielfalt der Kommunikation überhaupt.

Daß Englisch die Weltsprache ist, führt dazu, daß sie überall anders gesprochen wird. Das heißt, sie ist eine sehr flexible, bewegliche Sprache geworden: dein Englisch muß nicht mein Englisch sein, aber wir können uns trotzdem gut verständigen. Inzwischen kann man sogar von einer internationalen Sprache sprechen, die sich von ihren anglo-amerkanischen Würzeln losgelöst hat und sich an die Bedürfnisse der globalen Geschäftswelt angepaßt hat. Zunehmend hat man mit einer Geschäftssprache zu tun, die in Asien, Amerika und Europa verstanden wird, aber an keinem genauen Ort angesiedelt 
ist. Graddol schreibt von einem "polycentrism" und einer "destandardisation" der global benutzten englischen Sprache (Graddol, S. 56).

Zum Kern einer derart internationalen Sprache gehört eine tiefe Toleranz gegenüber abweichenden und gar wenn man nach Lehrbuch arbeitet - falschen Anwendungen. Sicherlich sollte man einen möglichst genauen Gebrauch der Sprache anstreben, was Grammatik und Lexik betrifft. Aber viel wichtiger ist es, mit soliden Grundkenntnissen zu arbeiten und überhaupt mit der Kommunikation anzufangen. Deutsche Politiker und andere Personen des öffentlichen Lebens haben in den allermeisten Fällen sehr gute Englischkenntnisse und treten sicher in internationalen Medien auf. Fehler machen sie aber fast alle, vor allem in der Anwendung englischer Zeitformen des Verbs. Das nimmt ihnen aber niemand übel; im Gegenteil: Sie werden respektiert, weil sie in der Lage sind, sich an Diskussionen zu anspruchsvollen Themen auf Englisch zu beteiligen. Viel interessanter als das Niveau ihrer Englischkenntnisse, sobald ein gutes Grundniveau erreicht ist, ist das, was sie sagen.

Deutsche Unternehmen können sich in einem internationalen Umfeld bewegen, in dem Englisch die Muttersprache ist. Viel häufiger aber werden sie dort arbeiten, wo Englisch nicht die Muttersprache ist, aber die Verkehrssprache. Wenn ein Betrieb einen Anruf aus Vietnam oder Italien entgegennimmt, werden in den meisten Fällen zwei Menschen miteinander sprechen, die Englisch als Fremdsprache gelernt haben, und sie werden Englisch benutzen. Sie haben verschiedene Erfahrungen mit dem Erlernen und der praktischen Anwendung der Fremdsprache und interessieren sich nicht mehr in erster Linie dafür, ob das, was sie sagen, einem abstrakten sprachlichen Regelwerk entspricht. Sie wollen und müssen möglichst schnell verstehen und verstanden werden. Ein wichtiges Ziel der praktischen Fremdsprachenausbildung ist, diese täglich notwendige Kommunikation zu fördern und zu trainieren.

\section{Die Sprachkompetenz von Unternehmen}

Die Anforderungen kleinerer und mittlerer Unternehmen im Land Brandenburg an fremdsprachlicher Kompetenz können in folgender Weise - verallgemeinernd - zusammengefaßt werden.

\subsection{Allgemeine Kommunikation}

a. Jedes Unternehmen, das im Ausland arbeiten will, sollte Mitarbeiter haben, die die Sprache der jeweiligen Länder zumindest soweit kennen, daß die oben beschriebenen Höflichkeiten des Alltags bewältigt werden können. Sicherlich wäre es erstrebenswert, über Kenntnisse der Muttersprache des Ziellands auf viel höherem Niveau zu verfügen. Das wird nicht immer sofort möglich sein; Englischkenntnisse sollten dann auf jeden Fall vorhanden sein. b. Jedes Unternehmen sollte Mitarbeiter haben, die gute Grundkenntnisse in Englisch haben, so daß sie in der Lage sind, spontan auf Anfragen zu reagieren und Gespräche allgemeinen Inhalts zu führen. Nichts ist peinlicher und schädlicher für ein Unternehmen, als wenn ein Interessierter deswegen nicht weiterkommt, weil niemand in der Lage war, mit ihm zu sprechen. Dabei geht es nicht nur um Englischkenntnisse, sondern um die Fähigkeit überhaupt zu kommunizieren. Small talk gehört in Deutschland - und besonders in Norddeutschland - nicht immer zu den Gepflogenheiten des Alltags, aber in einem englischsprachigen Umfeld kann seine Bedeutung nicht unterschätzt werden. Erkundigungen nach dem Wetter scheinen in Deutschland oft belanglos oder gelten als Zeitverschwendung. Im englischsprachigen Raum jedoch dient das oberflächliche Thema als Möglichkeit, sich mit dem Partner vertraut zu machen. Das kleine Gespräch liefert auch nebenbei viele Informationen hinsichtlich Herkunft und Bildungsstand. Dabei kann eine menschliche Nähe oder Sympathie entstehen. An fachlich und sprachlich besser qualifizierte Mitarbeiter kann man einen möglichen Partner immer später weiterleiten, aber bevor der Kontakt überhaupt so weit gediehen ist, muß das Gespräch gepflegt werden.

c. Jedes Unternehmen sollte Mitarbeiter haben, die die englische Sprache am Telefon benutzen können. Und alle Mitarbeiter, die das Telefon bedienen, sollten mindestens in der Lage sein, festzustellen, was der Anrufer will, und ihn auf Englisch weiterzuverbinden. Das ist gar nicht so schwer, denn es gehören nur einige leicht trainierbare Kenntnisse der Sprache des Telefonierens dazu. Wenn es um den ersten Eindruck des Unternehmens geht, ist es sicherlich besser, die Antwort "Hold the line, please" oder "I'll put you through, sir" als "Moment" oder gar Schweigen zu hören.

d. Jedes Unternehmen sollte Mitarbeiter haben, die Englisch lesen können. Dazu gehören Korrespondenz, Werbematerial, Zeitschriften und auch fachliches Material jeder Art. Damit dürften die meisten Betriebe weniger Probleme haben.

\subsection{Fachliche Kommunikation}

Bei der fachlichen Kommunikation wird jedes Unternehmen irgendwann auf eine Grenze stoßen, die nur mit professioneller Hilfe von Dolmetschern und Übersetzern zu überwinden ist. Diese Grenze genau zu kennen, spart Zeit und Geld. Sich selbst zu überschätzen ist gefährlich. Übersetzungen von Fach- und Werbetexten, die im Satzbau, in der Lexik und stilistisch so holprig und hölzern wirken wie eine unüberlegte wörtliche Übersetzung, begegnet man nicht selten bei kleineren wie bei großen und angesehenen Unternehmen.

a. Jedes Unternehmen sollte Mitarbeiter haben, die Anfragen jeder Art über organisatorische, betriebliche, produktbezogene, personelle und auch kulturelle Belange mündlich und schriftlich beantworten können. Das sollte sowohl in einem spontanen, informellen 
als auch in einem formellen Kontext möglich sein. Das heißt, daß Referate und Präsentationen über Produkte und Vorhaben vorbereitet werden müssen.

b. Es ist unerläßlich für die Selbstpräsentation, daß schriftliches Werbe- und Informationsmaterial vorliegt. Dazu gehören mittlerweile auch englische Seiten auf der Homepage im World Wide Web. Hier sollten in den allermeisten Fällen professionelle Übersetzer mitwirken, weil Fehler nicht nur einen schlechten Eindruck hinterlassen, sondern zu erheblichen Schwierigkeiten bei einem Geschäft führen können. Aber es sollte nicht dabei belassen sein, daß das Material außerhalb des Betriebs übersetzt wird. Sobald gutes Material vorhanden ist, sollten alle Mitarbeiter, die mit dem Ausland zu tun haben, sich damit beschäftigen und sich ihrer eigenen Fähigkeit, die Firma in der Fremdsprache zu vertreten, vergewissern. Ohne diese Rückwirkung von schriftlichem Material zu einem kommunikativen Umgang mit dem jeweiligen Thema hat sich die Investition in eine Übersetzung nur halb gelohnt.

c. Jedes Unternehmen wird Mitarbeiter benötigen, die Korrespondenz auf Englisch bewältigen können. Es gibt hierfür einige Standardbriefe und -formeln, die leicht variiert anwendbar sind. Und außerdem ist die Nachahmung erhaltener Korrespondenz oft ein verläßlicher Weg, eigene Fähigkeiten zu verbessern. Eine Website mit 100 Musterbriefen befindet sich unter: http://www.hut.fi/ rvilmi/LangHelp/writing/letters.

d. Wenn es zu tatsächlichen Geschäften mit ausländischen Unternehmen kommt und dann ins Detail bei größeren und komplizierteren Vorhaben geht, werden die meisten Betriebe für etwaige Verhandlungen Dolmetscher benutzen. Sich einzig und allein auf Dolmetscher zu verlassen, ist unserer Ansicht nach falsch. Im Idealfall ist der Dolmetscher nur eine Art notwendige Sicherheit für beide Parteien, die ohnehin das allermeiste verstehen, was gesagt oder geschrieben wird.

\section{Kulturelle Aspekte}

In Franz Kafkas Roman „Das Schloß“ geht der Landvermesser K. im Auftrag der Schloßherrn in ein unbenanntes Dorf, um eine Arbeit aufzunehmen. Doch K. scheitert an einem kulturellen Problem: Er versteht gar nichts von den Spielregeln einer ihm vollkommen fremden Welt. Die Befremdung, die in „Das Schloß“ abstrakt und damit absolut wirkt, hatte Kafka in seinem ersten Roman „Der Verschollene“ schon etwas konkreter beschrieben. Der junge Mann Karl Rossmann wandert nach Amerika aus und muß sich in der ungewohnten Umgebung fremder Manieren und einer ungewohnt harten Wirtschaft durchschlagen.

Kafkas Helden konnten sich nicht auf die Fremde vorbereiten, weil der Autor es ihnen nicht erlaubte. In dieser Lage muß und darf keiner sein, der im Ausland Geschäfte machen will. Zur Vorbereitung auf das Ausland kann man neben jeder finanziellen, rechtlichen oder andersgearteten wirtschaftlichen Information auch kulturelle Erfahrungen einholen.
Das ist aus zwei Gründen notwendig. Zum ersten sollten Umgangsformen in anderen Kulturen bekannt sein, um sicher auftreten zu können und vor etwaigen Fehlleistungen gefeit zu sein. Zweitens muß eine MarketingStrategie kulturelle Faktoren berücksichtigen. Dazu einige Beispiele:

Zum Umgang: Anredeformen unterscheiden in erheblichem Maße von Kultur zu Kultur, auch wenn alle Beteiligten die englische Sprache benutzen. Es entstehen Unsicherheiten, ob man in der jeweiligen Situation Voroder Familiennamen benutzt, ob man Titel verwendet und wie man die verschiedenen Menschen innerhalb einer betrieblichen Hierarchie anzusprechen hat. Vor allem der amerikanische und englische Gebrauch von Vornamen ist für viele Deutsche gewöhnungsbedürftig. Viele Ausländer dagegen verstehen nicht, wie deutsche Kollegen jahrelang zusammenarbeiten können und den Gebrauch der Sie-Form und von Familiennamen aufrechterhalten (vgl. Levine/Adelman, 1993, S. 241-79 für eine Diskussion US-amerikanischer Arbeitspraktiken und eventuelle kulturelle Probleme).

Wenn ein Inder Ihnen sagt, Sie können 'mal vorbeikommen, dann ist das oft eine Aufforderung, einen Termin zu vereinbaren. Wenn ein Engländer oder Amerikaner so etwas sagt, ist das selten mehr als eine allgemeine Floskel, oder genauer gesagt, ein Ausdruck seines allgemeinen Wohlwollens. Hier soll man die Botschaft so decodieren und entsprechend allgemein und unverbindlich reagieren: "That would be nice." Sofort nach einem Termin zu fragen,wäre nicht nur unhöflich, sondern macht deutlich, daß man die Botschaft nicht verstanden hat.

Wenn Sie in einer Kneipe mit Briten oder Iren sind, dann sollten Sie darauf achten, daß Getränke immer der Reihe nach für die ganze Runde gekauft werden. Engländer werden Sie nicht daran erinnern, aber sich dann ärgern, wenn Sie das vierte Mal ihre Runde verpaßt haben.

Mitarbeiter des amerikanischen Kommunikationsunternehmens AT + T mußten sich bei einem Projekt in Italien daran gewöhnen, daß immer beste Anzuige zu tragen sind, daß Meetings nicht pünktlich anfangen müssen und daß man im Restaurant nicht mehr über geschäftliche Fragen diskutiert (Cheryl Johnson und Carolyn S. Younker, in: Moran et. al., 1994, S. 179-185). Ein amerikanischer Autohersteller bereitete mit einem chinesischen Unternehmen ein Joint venture in China vor. Daß alles - aus amerikanischer Sicht - viel zu lange dauerte, war das Hauptproblem. Aber es stellte sich heraus, daß die chinesische Seite auch mit den - wieder aus amerikanischer Sicht - einfachsten Formulierungen im Vertrag Probleme hatte. Sie wollten den Inhalt des Wortes „etcetera“ genau ausgefuihrt haben; sie hatten keine Vorstellung davon, daß man bei finanziellen Vereinbarungen den Inflationsfaktor berücksichtigen sollte (Mary B. Teagarden, Mary Ann von Glinow, in: Moran et.al., 1994, S. 97-116). 
Zum Marketing: Die amerikanische Brauerei AnheuserBusch wollte sich mit ihrem Budweiser im deutschen und britischen Markt etablieren. Es wurden verschiedene Strategien entwickelt, den Trinkgewohnheiten in den beiden Ländern entsprechend. Kulturelle Informationen über deutsche und britische Kneipen wurden eingeholt, aber auch über die Mentalität: Gerade in Sachen Bier fand Anheuser-Busch heraus, daß die Briten und noch mehr die Deutschen äußerst konservativ sind. In Deutschland mußte das Bier in Flaschen verkauft werden, während es in England viel wichtiger wäre, in Kneipen Fuß zu fassen. Die Schwierigkeit, die darin bestand, neue europäische Kunden für ein Bierprodukt aus einem Land zu gewinnen, das nicht gerade für seine Bierkultur bekannt ist, war Anheuser-Busch bewußt. Der Versuch, das Bier über das Image des jungen, dynamischen USLifestyles zu verkaufen, scheiterte vorerst in Deutschland, hatte aber in England mehr Erfolg. Auch das wird kulturelle Gründe haben, die in der größeren Präsenz anderer imagebewußter amerikanischer Produkte (vor allem Levis) auf dem Markt zu suchen sind, aber auch in einer dynamischeren Jugendkultur in Kneipen, wo „exotische“ Biermarken schon im Trend lagen (John E. Walsh, Jr. und William P. Coon, in: Moran et. al., 1994. S. 241-255 und S. 256-266).

Ein Fernsehwerbespot für ein Putzmittel zeigte ein kleines Mädchen, das für ihren Bruder saubermacht. In Kanada wurde die Botschaft als sexistisch aufgefaßt (Hollet, 1993, S.46).

Eine Zeitungswerbung für eine Fluggesellschaft in Saudi Arabien zeigte eine Stewardess, die den glïicklichen Reisenden Champagner verabreichte. Alkohol ist illegal in Saudi Arabien, und Frauen ohne Schleier kommen nicht mit Männern zusammen (Hollet, 1993, S. 46).

Eine Werbung für Seifenpulver zeigte links einen Haufen dreckige Wäsche, das Produkt in der Mitte und saubere Kleider rechts. Die Werbekampagne scheiterte im Nahen Osten, weil man nicht beachtet hatte, daß dort von rechts nach links gelesen wird (Hollet, 1993, S. 46). Eine Fluggesellschaft mit dem Namen Emu wurde von australischen Kunden verschmäht. Der Vogel Emu kann nicht fliegen (Hollet, 1993, S. 46).

Das Wort „Kultur“ stammt aus dem Lateinischen und bedeutete ursprünglich „Pflege des Ackers“. Das heißt, daß in dem Wort eine andauernde Arbeit steckt, die ertragreich sein kann, und auch, daß das Wort eine Beziehung zu etwas anderem beinhaltet. Diese Arbeit sollte auf jeden Fall als Chance aufgefaßt werden, nicht nur geschäftlich. Eine andere Kultur kennenzulernen heißt auch, die eigene genauer $\mathrm{zu}$ betrachten und $\mathrm{zu}$ relativieren. Das Zeitempfinden, die Vorstellungen von Pünktlichkeit und Effizienz unterscheiden sich von Kultur zu Kultur ganz wesentlich. Wer in diesem Punkt nicht auf Überraschungen vorbereitet ist, wird es sicherlich erstmal schwer haben.

Aber wie kann man sich auf den Umgang mit der Fremde vorbereiten? Ein Teil der Marktforschung könnte als Kulturforschung gesehen werden. Man wird sicherlich versuchen, andere Betriebe oder Kollegen zu finden, die
Erfahrungen in dem Zielland haben. Am wichtigsten aber wird es immer sein, offen zu bleiben für Überraschungen, das Fremde nicht als Hindernis, sondern als Gewinn zu erleben, und neugierig zu sein. Vor Ort zu lernen ist immer der effektivste Weg, mit neuen Herausforderungen umzugehen. Die meisten Partner im Ausland werden bei der Klärung von Problemen helfen und das Interesse für ihr Leben und ihre Kultur wohlwollend aufnehmen. Schließlich haben auch ausländische Partner ein Interesse daran, mit ihren deutschen Kollegen auszukommen. Sie werden kaum so schweigsam oder konfus sein wie die Dorfbewohner in Kafkas Roman, die K. vergeblich um Hilfe bittet.

\section{Ziele der Fremdsprachenausbildung}

Die Fremdsprachenausbildung darf sich nicht auf die Vermittlung von rein sprachlichen Kenntnissen beschränken. Die alte Vorstellung, daß eine Sprache vorrangig über die Grammatik und über das Lesen gelernt wird, stammt ursprünglich aus der Methodik für klassische Sprachen, die auf moderne Fremdsprachen übertragen wurde. Ohne Grundkenntnisse der Grammatik kommt man nicht weit, aber selbst diese können im Sprechen gelernt werden. Wenn Grundkenntnisse vorhanden sind, sollte der Sprachunterricht ständig die Ziele im Auge haben, für die die Sprache ausgebildet wird.

Im besten Fall wird die Fremdsprachenausbildung für Wirtschaftsenglisch einer Art Kommunikationstraining gleichen, die zufällig in der Fremdsprache stattfindet. Dabei werden die Fähigkeiten vermittelt, die oben als die Bedürfnisse von Unternehmen angegeben wurden. Soziale Kommunikationsformen, das Telefonieren, die Korrespondenz, die mündliche und schriftliche Darstellung von Unternehmen und Produkten, die Entwicklung von fiktiven Werbe- oder Marketingstrategien, Verhandlungsführung, all das kann Bestandteil der Fremdsprachenausbildung sein. Das Klassenzimmer sollte zu einem Testfeld werden, in dem Individuen und Gruppen miteinander kommunizieren oder debattieren, ihre Projekte einander vorstellen, formelle wie informelle Präsentationen üben, Fallstudien diskutieren und vor allem praxisbezogene Themen behandeln. Rhetorische Fähigkeiten stehen im Vordergrund. Das spezifische Vokabular des Wirtschaftsenglisch wird dann zwangsläufig zum Inhalt eines lebendigen Lernprozesses.

Wichtiger als jedes Fachvokabular bleibt aber die Kommunikation. Der Sprachunterricht sollte Selbstbewußtsein im aktiven Gebrauch der Fremdsprache fördern und die Hemmungen sowie die passive Lernhaltung abbauen, mit denen viele Schulabgänger leider behaftet sind. Ein wirtschaftliches oder technisches Fachvokabular wird man sich immer dann schnell und gezielter aneig. nen, wenn man es im Betrieb braucht, solange eine solide Kommunikationsfáhigkeit vorhanden ist.

Das Material, das im Fremdsprachenunterricht benutzt wird, kann in vielen Fällen originalen Medien jeder Art 
entnommen sein. Ein Mangel an Angeboten besteht nicht: Das Fernsehen, der Rundfunk, Zeitungen und Zeitschriften, Werbematerial und betriebliches Informationsmaterial jeder Art sind alle auf Englisch leicht zu bekommen. Auch an guten Lehrwerken mangelt es nicht. Das Fach „Englisch als Fremdsprache“ ist zu einer internationalen Industrie geworden, die den Schwerpunkt immer mehr auf praxisorientierte Kommunikation legt. Und es ist zu erwarten, daß in der Zukunft dieses Fach nicht von englischsprachigen Muttersprachlern beherrscht wird. Die Bedürfnisse in einzelnen Länder und die wachsende weltweite Zahl von Menschen, für die Englisch die zweite Sprache ist, werden es bedingen, daß sich das globale Englisch in einer wirklich polyphonen Umgebung entwickelt, wo die Sprache den jeweiligen lokalen Gegebenheiten angepaßt ist.

Von den vielfältigen Angeboten, die uns täglich auf Englisch gegeben werden, können auch Mitarbeiter in Betrieben Gebrauch machen, die nicht mehr an einem Sprachkurs teilnehmen. Das reicht von der Lektüre von Zeitungen bis hin zum gezielten oder spielerischen Umgang mit dem Internet.

Um die sprachlichen und kulturellen Probleme zu bewältigen, die das Ausland mit sich bringt, brauchen Unternehmen nicht nur Mitarbeiter mit guten Sprach- und kulturellen Kenntnissen. Sie brauchen auch das, was heutzutage zu einem der Schlagwörter schlechthin in der Wirtschaft geworden ist: Flexibilität. Das bedeutet nicht nur räumliche Flexibilität oder Mobilität, sondern auch geistige. Auch da kann das Erlernen einer Sprache und die Gestaltung des Fremdsprachenunterrichts Einfluß nehmen. Eine andere Sprache zu lernen, heißt immer, die eigenen Perspektiven zu erweitern, mit anderen Denkweisen konfrontiert zu werden und diese kritisch aufzunehmen. Und wenn der Sprachunterricht so gestaltet werden kann, daß er im weitesten Sinne auf Problemlösung und rhetorischem Training basiert, dann kann er ein Teil einer gesamten Ausbildung sein, in der eine kreative und kritische Haltung den gleichen Stellenwert hat wie die reine Stoffvermittlung. Das ist der Idealfall, der nicht immer gelingen wird, aber wenn er gelingt, dann kann das Ergebnis in der Pflege vor allem einer Eigenschaft bestehen: Neugierde.

\section{Literatur}

David Crystal: The Cambridge Encyclopedia of The English Language, Cambridge, The Press Syndicate of the University of Cambridge, 1995.

David Crystal, English as a Global Language, Cambridge, Cambridge University Press, 1997.

David Graddol, The Future of English? London, The British Council, 1997.

Vicki Hollet, Business Objectives, Oxford, Oxford University Press, 1993.

Deena R. Levine, Mara B. Adelman, Beyond Language, Cross-Cultural Communication, New Jersey, Regents/ Prentice Hall, zweite Aufl., 1993.
Robert T. Moran, David O.Braaten, John E. Walsh, Jr, Hg, International Business Case Studies For the Multicultural Marketplace, Houston, London, Paris, Zürich, Tokyo, Gulf Publishing Company, 1994.

Bernd Wonneberger, Hans Könecke, Zur Wettbewerbsfähigkeit von Brandenburger KMU auf den Märkten in Ost- und Südostasien. Tagungsbeitrag zum Wildauer Workshop am 8.10.1997.

"Language and Electronics. The Coming Global Tongue", The Economist, 21.12.1996-3.1.1997, S. 45-48.

"A Gift of Tongues", The Economist, 18.-24.10.1997, S. 107-08.

\section{Websites}

The British Council (English worldwide): http://www.britcoun.org/index.htm The English Company (news and newsletter on developments in English):

http://www.english.co.uk/FOE/contents/html

Websites mit sehr guten, vielfältigen Links zu englischsprachigen Materialien jeder Art:

http://userpage.fu-berlin.de/ tanguay/earn_english.htm http://www.pacificnet.net/ sperling

http://www.chs.chico.k12.ca.us/libr/webres/esl.html

\section{Korrespondenz:}

http://www.hut.fi/ rvilmi/LangHelp/writing/letters

\section{Berlin auf Englisch:}

http://www.berlin.de/cgi-bin/insert_referer.pl/english/ Eabout/index.html

\section{Verfasser}

\section{Dr. Greg Bond}

Technische Fachhochschule Wildau

Sprachenzentrum

Bahnhofstraße

15745 Wildau

E-mail: bond@sprz.tfh-wildau.de

Tel. +49 (0) 3375 - 508367

\section{John O'Donoghue, M.A.}

Technische Fachhochschule Wildau

Sprachenzentrum

Bahnhofstraße

15745 Wildau

E-mail: odonjohn@sprz.tfh-wildau.de

Tel. +49 (0) 3375 - 508367 